USACH-FM-00/01

PM- $00 / 01$

\title{
Cubic root of Klein-Gordon equation
}

\author{
Mikhail S. Plyushchay ${ }^{* a, b}$ and Michel Rausch de Traubenberg ${ }^{\dagger c, d}$ \\ ${ }^{a}$ Departamento de Física, Universidad de Santiago de Chile, Casilla 307, Santiago 2, Chile \\ ${ }^{b}$ Institute for High Energy Physics, Protvino, Russia \\ ${ }^{c}$ Laboratoire de Physique Mathématique, Université Montpellier II, \\ Place E. Bataillon, 34095 Montpellier, France \\ ${ }^{d}$ Laboratoire de Physique Théorique, 3 rue de l'Université, 67084 Strasbourg, France
}

\begin{abstract}
We construct new relativistic linear differential equation in $d$ dimensions generalizing Dirac equation by employing the Clifford algebra of the cubic polynomial associated to Klein-Gordon operator multiplied by the mass parameter. Unlike the Dirac case where the spin content is unique and Lorentz covariance is manifest, here the spin as well as Lorentz covariance of the theory are related to the choice of representation of the Clifford algebra. One of the considered explicit matrix representations gives rise to anyon-like fields in $d=1+1$. Coupling to a $\mathrm{U}(1)$ gauge field is discussed and compared with Dirac theory.
\end{abstract}

*e-mail: mplyushc@lauca.usach.cl

†e-mail: rausch@lpm.univ-montp2.fr 


\section{Introduction}

A possible way to obtain relativistic wave equations is to relate the spin degrees of freedom to an appropriate algebraic structure. The most famous example is the Dirac equation for spin $1 / 2$ particles in which spin is associated to Clifford algebra. Beyond this simplest example several possibilities have been considered, as for instance the Duffin-Kemmer-Petiau (DKP) algebra relevant for a first order equation for a spin zero and spin one particles [1]. These two types of algebra (Dirac and DKP) appear as special cases of the parafermionic algebra of order $p(p=1$ being the Clifford algebra and $p=2$ the DKP one) [2]. The parafermionic algebra is defined by cubic relations, encoding the basic property that the Lorentz generators are just given by the commutator of the generators of the algebra itself. Besides these algebraic descriptions for integer and half-integer spin states in any space-time dimension, specific properties appear in low dimensions. For instance, in $2+1$ dimensions anyons are realized on infinite dimensional representations of the universal covering group of the $(2+1)$-dimensional Lorentz group [3, 迎. Relativistic equations for a relativistic anyon are more involved, and various possibilities have been considered [5, 6]. In particular, the (super)algebraic structure of the so called deformed Heisenberg algebra with reflection related to parabosons and parafermions [7] allowed one of us to construct linear differential equations describing universally anyons and ordinary fields of integer and half-integer spin [8], including topologically massive vector gauge field [9].

In this paper, we consider an alternative way for obtaining a relativistic wave equation by simple extension of Dirac's idea. We just write the Klein-Gordon operator as a perfect $n$-th power (here we study the simplest case $n=3, i$. e. we introduce an operator $D$ such that $\left.D^{3}=-m\left(\square^{2}+m^{2}\right)\right)$. The present approach is based on writing the quadratic form associated to the Minkowski metric as a cubic form. A set of generators $g_{\mu}$ and $\tilde{g}$ associated, in turn, to the momentum vector $p_{\mu}$ and to the mass parameter $m$ is then introduced. This allows us to define a first order relativistic equation of the form $\left(i g^{\mu} \partial_{\mu}+m \tilde{g}\right) \psi=0$, which can be treated as a special type of a generic Dirac-like equations 10 ]

$$
\left(i \beta^{\mu} \partial_{\mu}+m \tilde{\beta}\right) \psi=0 .
$$

The basic algebra (generated by $g_{\mu}$ and $\tilde{g}$ ) giving rise to such a linearization is the so called Clifford algebra of polynomials [11]. This is an $n$-th order algebra where the generators are submitted to $n$-th order constraints. This infinite dimensional algebra admits several finite dimensional representations allowing us to define a first order wave equation. However, although the algebra itself is Lorentz-invariant, the covariance of the relativistic wave equation is not guaranteed for a given representation. In other words, there is no guarantee to construct Lorentz generators in terms of the $g$ 's themselves. This is very different to the parafermionic situation. Various explicit types of manifestly Lorentz covariant finite dimensional representations of the cubic Clifford algebras can be obtained and compared with the spinorial construction of the Lorentz group (related to ordinary Clifford algebras). On the other hand, no infinite dimensional representations of cubic Clifford algebras are known (even though these algebras are infinite dimensional). In the case of a manifestly covariant equation, the approach gives us the possibility to relate the spin degree of freedom with a new algebraic structure. At the same time, some results concerning the cubic Clifford algebra and associated relativistic wave equation can be established independently of the representation we select (or independently of the content of the relativistic field).

The paper is organized as follows. In section 2, we study the algebraic structure with which

the Klein-Gordon operator is written through a perfect cube. Some conditions are then given in order to ensure a covariant relativistic wave equation. Two types of matrix representations are given: one with a manifest $d$-dimensional Lorentz covariance ( $d$ is arbitrary), and another with 
no Lorentz covariance in $2+1$ dimensions but admitting covariance in $1+1$ dimensions. Section 3 is devoted to the study of the relativistic equation itself. Its solutions are given by means of appropriate projectors. Requirement of covariance of the equation gives us an alternative way to calculate Lorentz generators. In $1+1$ dimensions our approach gives a new relativistic anyon-like wave equation for fields of spin $1 / 3$ and $-2 / 3$ by means of $9 \times 9$ matrices. We discuss also coupling to a U(1) gauge field and observe that new structures appear here in comparison with Dirac theory. Section 4 contains some conclusions and comments.

\section{Cubic root of Klein-Gordon operator}

\subsection{Clifford algebra of the polynomial}

The Clifford algebra associated with Dirac equation is related to the linearization of the quadratic polynomial $P_{2}(x)=\left(x^{0}\right)^{2}-\left(x^{1}\right)^{2}-\cdots-\left(x^{d-1}\right)^{2}$. In a similar way there appear the so-called Clifford algebras of polynomials [11]. Such algebras can naturally be defined by introducing a series of generators leading to a linearization of a given polynomial of degree $n$ with $k$ variables. From the isomorphism between degree $n$ polynomials and symmetric tensors of order $n$, one can write any homogeneous polynomial $P$ as

$$
P\left(x_{1}, \cdots, x_{k}\right)=\sum_{\{i\}=1}^{k} x_{i_{1}} x_{i_{2}} \cdots x_{i_{n}} g_{i_{1} \cdots i_{n}}
$$

with $g_{i_{1} \cdots i_{n}}$ being the associated symmetric rank $n$ tensor. As for the ordinary quadratic Clifford algebra, writing the polynomial $P$ as a perfect power of $n$ defines the Clifford algebra $\mathcal{C}_{P}$ :

$$
P\left(x_{1}, \cdots, x_{k}\right)=\sum_{\{i\}=1}^{k} x_{i_{1}} x_{i_{2}} \cdots x_{i_{n}} g_{i_{1} \cdots i_{n}}=\left(x_{1} g_{1}+\cdots+x_{k} g_{k}\right)^{n},
$$

where the generators $g_{1}, \cdots, g_{k}$ are associated with the variables $x_{1}, \cdots, x_{k}$. Developing explicitly the $n$-th power and identifying all the terms leads to the relations

$$
S_{n}\left(g_{i_{1}}, \cdots, g_{i_{n}}\right):=\frac{1}{n !} \sum_{\sigma \in \Sigma_{n}} g_{i_{\sigma(1)}} \cdots g_{i_{\sigma(n)}}=g_{i_{i} \cdots i_{n}}
$$

with $\Sigma_{n}$ the symmetric group of order $n$. The Clifford algebra of the polynomial $P$ is then the order $n$ algebra generated by the $g_{i}$ submitted to the constraints (2.2).

The algebra (2.2) is very different from the usual Clifford algebra. Indeed, $\mathcal{C}_{P}$ is defined through $n$-th order constraints, and consequently the number of independent monomials increases with

polynomial's degree (for instance, $g_{1}^{2} g_{2}$ and $g_{1} g_{2} g_{1}$ are independent). This means that we do not have enough constraints among the generators to order them in some fixed way and, as a consequence, $\mathcal{C}_{P}$ turns out to be an infinite dimensional algebra. However, it has been proved that for any polynomial a finite dimensional (non-faithful) representation can be obtained [12]. But, for polynomial of degree higher than two, we do not have a unique representation, and inequivalent representations of $\mathcal{C}_{P}$ (even of the same dimension) can be constructed (see, for instance, [13] and below for the special cubic polynomials). Furthermore, the problem of classification of the representations of $\mathcal{C}_{P}$ is still open, though it has been proved that the dimension of the representation is a multiple of the degree of the polynomial [14]. For more details one can see [15] and references therein. 


\subsection{Clifford algebra of the cubic root of Klein-Gordon operator}

With the described Clifford algebras $\mathcal{C}_{P}$ one can represent the massive Klein-Gordon operator (in any $d$-dimensional space-time) as a perfect $n$-th power. This can be achieved with the help of Clifford algebra of the polynomial $P_{n, a}(p)=m^{n-2 a}\left(p^{\mu} p_{\mu}-m^{2}\right)^{a}=\left(p_{\mu} g^{\mu}+m \tilde{g}\right)^{n}, n \geq 2 a$, where $\eta_{\mu \nu}=\operatorname{diag}(1,-1, \cdots,-1)$ is the Minkowski metric and $m$ is a mass parameter. Here we investigate the case $n=3$ allowing us to take a cubic root of Klein-Gordon operator via the relation $m\left(p^{\mu} p_{\mu}-m^{2}\right)=\left(p_{\mu} g^{\mu}+m \tilde{g}\right)^{3}$. In this 3-root case, the generators $g_{\mu}$ and $\tilde{g}$ satisfy the cubic algebra

$$
S_{3}(\tilde{g}, \tilde{g}, \tilde{g})=\tilde{g}^{3}=-1, \quad S_{3}\left(g_{\mu}, \tilde{g}, \tilde{g}\right)=0, \quad S_{3}\left(g_{\mu}, g_{\nu}, \tilde{g}\right)=\frac{1}{3} \eta_{\mu \nu}, \quad S_{3}\left(g_{\mu}, g_{\nu}, g_{\lambda}\right)=0 .
$$

We denote $\langle g\rangle$ the Clifford algebra generated by the $g$ 's submitted to the constraints (2.3). Obviously, the product

$$
S O(d-1,1) \times \mathbb{Z}_{2} \times \mathbb{Z}_{2} \times \mathbb{Z}_{3}
$$

is an outer automorphism of this algebra. With respect to $S O(d-1,1), g_{\mu}(\tilde{g})$ are in the vector (scalar) representation. The two $\mathbb{Z}_{2}$ factors are due to the $P$ (parity) and $T$ (time reversal) invariance of (2.3). The $\mathbb{Z}_{3}$ automorphism is associated with the substitution $\left(g_{\mu}, \tilde{g}\right) \longrightarrow\left(q g_{\mu}, q \tilde{g}\right)$ with $q$ a cubic root of the unity, $q^{3}=1$. In other words the algebra (2.3) admits a $\mathbb{Z}_{3}$-graded structure and the $g$ 's have a gradation one.

From the generators of the algebra one can construct the following 0-grade vectors,

$$
\Gamma_{\mu}=\tilde{g}^{2} g_{\mu}, \quad \tilde{\Gamma}_{\mu}=g_{\mu} \tilde{g}^{2}, \quad \hat{\Gamma}_{\mu}=\tilde{g} g_{\mu} \tilde{g}
$$

Due to the algebra (2.3), these operators are the simplest vectors of gradation zero, from which more complicated 0-grade vectors can be constructed by inserting into them 0-grade products of three generators $g_{\lambda}, g^{\lambda}$ and $\tilde{g}$ (with summation in $\lambda$ implied). The second equation from (2.3) means, however, the linear dependence of the vectors (2.5):

$$
\Gamma_{\mu}+\tilde{\Gamma}_{\mu}+\hat{\Gamma}_{\mu}=0 .
$$

A natural question we should address is whether the automorphisms (2.4) are inner automorphisms. When we have an inner automorphism, this enables us to write down the Lorentz transformations (specified by the matrix $\Lambda$ ) as $\Lambda^{\mu}{ }_{\nu} g^{\nu}=S(\Lambda) g^{\mu} S^{-1}(\Lambda)$ and $\tilde{g}=S(\Lambda) \tilde{g} S^{-1}(\Lambda)$ with $S(\Lambda) \in<g>$. At the infinitesimal level this is reduced to the possibility to find the generators $J_{\mu \nu}$ such that

$$
\left[J_{\mu \nu}, g_{\alpha}\right]=i\left(\eta_{\mu \alpha} g_{\nu}-\eta_{\nu \alpha} g_{\mu}\right), \quad\left[J_{\mu \nu}, \tilde{g}\right]=0,
$$

with $J_{\mu \nu}$ constructed in terms of the $g$ 's. From the requirement that $J_{\mu \nu}$ is a grade-zero antisymmetric rank 2 tensor, due to Eq. (2.7) it is constructed only with one $g_{\mu}$, one $g_{\nu}$ and scalars. The only scalar we have aref $\tilde{g}$ and $g^{\lambda} g_{\lambda}$. If $J_{\mu \nu}$ does not contain the contracted product(s) $g^{\lambda} g_{\lambda}$ (there is a strong argument in this direction, see Eq. (2.9) or Eq. (3.12), next section), then in terms of zero-grade vectors (2.5) one can construct only two possible generators commuting with $\tilde{g}$,

$$
\begin{aligned}
& J_{\mu \nu}^{(1)}=\Gamma_{\mu} \Gamma_{\nu}+\tilde{\Gamma}_{\mu} \tilde{\Gamma}_{\nu}+\tilde{\Gamma}_{\mu} \Gamma_{\nu}=\tilde{g} g_{\mu} g_{\nu}+g_{\mu} \tilde{g} g_{\nu}+g_{\mu} g_{\nu} \tilde{g}, \\
& J_{\mu \nu}^{(2)}=\Gamma_{\mu} \Gamma_{\nu}+\tilde{\Gamma}_{\mu} \tilde{\Gamma}_{\nu}+\Gamma_{\mu} \tilde{\Gamma}_{\nu}=\tilde{g}^{2} g_{\mu} \tilde{g}^{2} g_{\nu}+g_{\mu} \tilde{g}^{2} g_{\nu} \tilde{g}^{2}+\tilde{g}^{2} g_{\mu} g_{\nu} \tilde{g}^{2},
\end{aligned}
$$

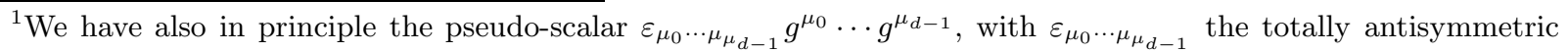
tensor of rank $d$.
} 
where $\mu \neq \nu$ assumed. To obtain the expression of $J_{\mu \nu}^{(1)}$ we have used (2.3), and it is easy to see that $J_{\mu \nu}^{(1)}$ in (2.8) are antisymmetric in $\mu$ and $\nu$ (see the second equation in (2.3)). Using Eq. (2.3), we get

$$
\left[J_{\mu \nu}^{(1)}, g_{\alpha}\right]=2\left(\eta_{\mu \alpha} g_{\nu}-\eta_{\nu \alpha} g_{\mu}\right)-\left[g_{\mu} g_{\nu} g_{\alpha}+g_{\mu} g_{\alpha} g_{\nu}+g_{\alpha} g_{\mu} g_{\nu}, \tilde{g}\right], \quad\left[J_{\mu \nu}^{(1)}, \tilde{g}\right]=0 .
$$

One could directly calculate $\left[J_{\mu \nu}^{(2)}, g_{\alpha}\right]$ to find that $J_{\mu \nu}^{(2)}$ does not fulfill either the correct commutation relations with $g_{\alpha}$ (we already know that $\left[J_{\mu \nu}^{(2)}, \tilde{g}\right]=0$ ). Instead, it is more easy to check this on explicit example (see the $9 \times 9$ matrix representation hereafter (2.12)). So, neither $J_{\mu \nu}^{(1)}$ nor $\left(J_{\mu \nu}^{(2)}-J_{\nu \mu}^{(2)}\right)$ (the antisymmetrized version of $J_{\mu \nu}^{(2)}$ ) could be the Lorentz generators. However, if the second part of the commutator of $J_{\mu \nu}^{(1)}$ with $g_{\alpha}$ in (2.9) vanishes, then $J_{\mu \nu}^{(1)}$ are the Lorentz generators (as it is the case for the representation (2.11), see below). Conversely, if we assume that one can find $J_{\mu \nu}$ satisfying (2.7), it is tempting to conclude that this implies vanishing the second part of the commutator of $J_{\mu \nu}^{(1)}$ with $g_{\alpha}$ (see Eq. (2.9)). Indeed, if we introduce $J_{\mu \nu}$ as a 0 -grade operator constructed with one $g_{\mu}$, one $g_{\nu}$ and the scalars $\tilde{g}$ and $g^{\lambda} g_{\lambda}$, from the Lorentz covariance we find that $J_{\mu \nu}^{(1)}$ transforms as a rank 2 tensor. So, $\left[J_{\mu \nu}, J_{\alpha \beta}^{(1)}\right]$ has a simple form. But now, if using (2.9) we calculate $\left[J_{\mu \nu}^{(1)}, J_{\alpha \beta}\right]$, this gives a very complicated expression (coming from $\left[J_{\mu \nu}^{(1)}, g^{\lambda} \ldots g_{\lambda}\right]$ ), and the cancellation seems to be difficult to obtain except if $\left[g_{\mu} g_{\nu} g_{\alpha}+g_{\mu} g_{\alpha} g_{\nu}+g_{\alpha} g_{\mu} g_{\nu}, \tilde{g}\right]=0$. In such a case $J_{\mu \nu}$ is equal to $\frac{i}{2} J_{\mu \nu}^{(1)}$. Furthermore, the results of section 2 also suggest that $J_{\mu \nu}$ does not contain $g^{\lambda} \ldots g_{\lambda}$.

Consequently, we could conjecture that the outer automorphism $S O(d-1,1)$ becomes an inner automorphism if and only if $\left[g_{\mu} g_{\nu} g_{\alpha}+g_{\mu} g_{\alpha} g_{\nu}+g_{\alpha} g_{\mu} g_{\nu}, \tilde{g}\right]=0$, and then we have

$$
J_{\mu \nu}=\frac{i}{4}\left(\tilde{g} g_{[\mu} g_{\nu]}+g_{[\mu} \tilde{g} g_{\nu]}+g_{[\mu} g_{\nu]} \tilde{g}\right)
$$

with $g_{[\mu} g_{\nu]}=g_{\mu} g_{\nu}-g_{\nu} g_{\mu}$.

So, on the one hand, for a given representation $\mathcal{R}_{g}$ of $\left\langle g>, g_{\mu}, \tilde{g} \longrightarrow G_{\mu}, \tilde{G}\right.$, there is no guarantee to find the generators $J_{\mu \nu}$ constructed from the $g$ 's. In such a case the representations $\mathcal{R}_{g}=<G_{\mu}, \tilde{G}>$ and $\mathcal{R}_{g}^{\Lambda}=<\Lambda^{\mu}{ }_{\nu} G^{\nu}, \tilde{G}>$ are inequivalent. This is very different to the usual Clifford algebra casef. But, on the other hand, if $S O(d-1,1)$ is an inner automorphism, this means that $\mathcal{R}_{g}=<G_{\mu}, \tilde{G}>$ and $\mathcal{R}_{g}^{\Lambda}=<\Lambda^{\mu}{ }_{\nu} G^{\nu}, \tilde{G}>$ are equivalent representations, or that the $G$ 's act on an appropriate representation of the Lorentz group. This constitute an adapted extension of the spinorial construction of the Lorentz group (related to the cubic instead of quadratic Clifford algebras).

\subsection{Examples of $<g>$-representations}

As we noted above, representations of the Clifford algebras of polynomials are not classified and only some special matrix representation are known. For $\langle g\rangle$ we have found two types of representations. The first one is constructed with the usual Dirac matrices in any space-time dimension,

$$
G_{\mu}=\left(\begin{array}{ccc}
0 & \gamma_{\mu} & 0 \\
0 & 0 & \gamma_{\mu} \\
0 & 0 & 0
\end{array}\right), \quad \tilde{G}=\left(\begin{array}{ccc}
0 & i \gamma_{d+1} & 0 \\
0 & 0 & i \gamma_{d+1} \\
1 & 0 & 0
\end{array}\right)
$$

\footnotetext{
${ }^{2}$ However, when the space-time dimension is odd, we have two inequivalent representations (equivalent to $\pm \gamma_{\mu}$ with $\gamma_{\mu}$ the Dirac matrices) because there is no $\gamma_{5}$-like matrix (chirality). Thus, the $T$ inversion is an outer automorphism for the usual Clifford algebra in odd dimensions.
} 
Because of the presence of the matrix $\gamma_{d+1}$, in odd-dimensional space-time we assume that $\gamma_{\mu}$ are realized via the gamma-matrices $\gamma_{\mu}^{(d-1)}$ corresponding to dimension $(d-1)$, e.g., in the form $\gamma_{\mu}=\gamma_{\mu}^{(d-1)} \otimes \sigma_{1}$, whereas $\gamma_{d+1}$ can be chosen as $\gamma_{d+1}=1 \otimes \sigma_{3}$. It is easy to see that in this Dirac-like representation both $J_{\mu \nu}^{(1)}$ and $J_{\mu \nu}^{(2)}$ in Eq. (2.8) give $J_{\mu \nu}=-\frac{i}{2} \gamma_{\mu} \gamma_{\nu} \otimes \operatorname{diag}(1,1,1)$.

The second representation is obtained by linearizing firstly the polynomial $m\left(\left(p_{0}\right)^{2}-\left(p_{1}\right)^{2}-\cdots\right)$,

$$
m\left(\left(p_{0}\right)^{2}-\left(p_{1}\right)^{2}-\cdots\right)=\left(\begin{array}{ccc}
0 & m & 0 \\
0 & 0 & p_{0}+p_{1} \\
p_{0}-p_{1} & 0 & 0
\end{array}\right)^{3}+\cdots
$$

with subsequent linearization of this sum of perfect cubes by means of the twisted tensorial product [13. As a result, we end up with the $3^{\left[\frac{d+2}{2}\right]} \times 3^{\left[\frac{d+2}{2}\right]}$-dimensional matrices, with $[a]$ being the integer part of $a$. In $2+1$ dimensions, the corresponding representation is given by the $9 \times 9$ matrices:

$$
G_{\rho}=\left(\begin{array}{ccc}
T_{\rho} & \mathbf{0} & \mathbf{0} \\
\mathbf{0} & q T_{\rho} & \mathbf{0} \\
\mathbf{0} & \mathbf{0} & q^{2} T_{\rho}
\end{array}\right), \rho=0,1, G_{2}=\left(\begin{array}{ccc}
\mathbf{0} & \mathbf{0} & \mathbf{0} \\
\mathbf{0} & \mathbf{0} & I \\
I & \mathbf{0} & \mathbf{0}
\end{array}\right), \tilde{G}=\left(\begin{array}{ccc}
\tilde{T} & -I & \mathbf{0} \\
\mathbf{0} & q \tilde{T} & i I \\
-i I & \mathbf{0} & q^{2} \tilde{T}
\end{array}\right),
$$

where $q$ is a primitive cubic root of the unity, $\mathbf{0}$ and $I$ are zero and unit $3 \times 3$ matrices, and

$$
T_{0}=\left(\begin{array}{ccc}
0 & 0 & 0 \\
0 & 0 & 1 \\
1 & 0 & 0
\end{array}\right), \quad T_{1}=\left(\begin{array}{ccc}
0 & 0 & 0 \\
0 & 0 & -1 \\
1 & 0 & 0
\end{array}\right), \quad \tilde{T}=\left(\begin{array}{ccc}
0 & 1 & 0 \\
0 & 0 & 0 \\
0 & 0 & 0
\end{array}\right)
$$

On this explicit representation, one can check that $J_{\mu \nu}^{(1)}$ and $\left(J_{\mu \nu}^{(2)}-J_{\nu \mu}^{(2)}\right)$ defined by (2.8) do not satisfy the correct commutation relation with $G_{\alpha}$. Moreover, one can also directly check that there does not exist any $9 \times 9$ matrix $J_{\mu \nu}$ satisfying (2.7). Therefore, for this representation $S O(2,1)$ is an outer automorphism.

Representation (2.12) turns out to be more interesting in the case of $1+1$ dimensions. Indeed, the matrices (2.12) lead to different types of $9 \times 9$ dimensional representations. The first type is given by the set of generators $\mathcal{R}_{02}=<G_{0}, G_{2}, \tilde{G}>$ or by the set $\mathcal{R}_{12}=<e^{i \pi / 3} G_{1}, G_{2}, \tilde{G}>$. For both these representations, there is no Lorentz generator satisfying Eq. (2.7). The second type representation is characterized by the set

$$
\mathcal{R}_{01}=<G_{0}, G_{1}, \tilde{G}>,
$$

and by the Lorentz generator

$$
J_{01}=\frac{i}{3}\left(\begin{array}{ccc}
1 & 0 & 0 \\
0 & 1 & 0 \\
0 & 0 & -2
\end{array}\right) \otimes\left(\begin{array}{ccc}
1 & 0 & 0 \\
0 & 1 & 0 \\
0 & 0 & 1
\end{array}\right)
$$

which satisfies the correct commutation relations with $G_{0}, G_{1}, \tilde{G}$, i.e. for this representation the automorphism $S O(1,1)$ is internal. Traceless generator 2.15) is obtained via Eq. (2.10) with appropriate 'renormalization' consisting in a shift for the matrix proportional to the unit one.

Finally, let us note that in $0+1$ dimension, there exists the $3 \times 3$ representation of $\langle g\rangle$ given by the matrices

$$
G_{0}=\left(\begin{array}{ccc}
0 & 1 & 0 \\
0 & 0 & 1 \\
0 & 0 & 0
\end{array}\right), \quad \tilde{G}=\left(\begin{array}{ccc}
0 & 1 & 0 \\
0 & 0 & -1 \\
1 & 0 & 0
\end{array}\right)
$$


To conclude the discussion of the representations of the Clifford algebra $\langle g\rangle$, one notes that only (some) finite dimensional representations of Clifford algebras have been found. On the other hand, as we noted above, in $2+1$ dimensions anyons are related to infinite dimensional representation of $\overline{S L(2, \mathbb{R})}$ (the universal covering of Lorentz group) [3, 4]. So, it would be very interesting to try to find infinite dimensional representations of $\langle g\rangle$ in relation to the infinite dimensional representation of $\overline{S L(2, \mathbb{R})}$.

\section{Cubic root of Klein-Gordon equation}

\subsection{First order equations and almost Dirac algebras}

After the formal discussion of the algebra $\langle g\rangle$ and its representations, now we are ready to define an adapted extension of the Dirac operator being a cubic root, instead of a quadratic one, of the Klein-Gordon operator,

$$
D=\left(i g^{\mu} \partial_{\mu}+m \tilde{g}\right), \quad D^{3}=-m\left(\square^{2}+m^{2}\right),
$$

with the $g$ 's defined in the previous section. This operator enables us to define two possible equations generalizing the Dirac one and its conjugate,

$$
\left(i g^{\mu} \partial_{\mu}+m \tilde{g}\right) \psi=0, \quad \bar{\psi}\left(i \overleftarrow{\partial_{\mu}} g^{\mu}+m \tilde{g}\right)=0
$$

where $\bar{\psi} \overleftarrow{\partial_{\mu}} \equiv \partial_{\mu} \bar{\psi}$. Following the general discussion of the previous section, if for a given representation of $\langle g\rangle$ the outer automorphism $S O(d-1,1)$ is promoted to an inner one, then the fields $\psi$ and $\bar{\psi}$ turn out to be in some representation $\mathcal{D}$ and $\mathcal{D}^{\star}$ (the dual representation of $\mathcal{D}$ ) of $\overline{S O(d-1,1)}$. In other words, under a Lorentz transformation $\Lambda$ the fields transform as $S(\Lambda) \psi$ and $\bar{\psi} S^{-1}(\Lambda)$. This means that the equations (3.2) constitute alternative relativistic equations. In the opposite case, (3.2) has no Lorentz covariance. Some general results upon this equation can be established without specifying concrete representation for $\langle g\rangle$. Similar equations already appeared in [16] in the world-line formalism (for the massless case) in the context of fractional supersymmetry [17].

If we multiply the first equation by $\tilde{g}^{2}$ on the left and the second on the right, we get

$$
\left(i \Gamma^{\mu} \partial_{\mu}-m\right) \psi=0, \quad \bar{\psi}\left(i \overleftarrow{\partial_{\mu}} \tilde{\Gamma}^{\mu}-m\right)=0
$$

with the matrices $\Gamma_{\mu}$ and $\tilde{\Gamma}_{\mu}$ defined in (2.5). A direct calculation with the algebra (2.3) leads to the almost Dirac algebras

$$
\left\{\Gamma_{\mu}, \Gamma_{\nu}\right\}=2 \eta_{\mu \nu}-\left\{g_{\mu}, g_{\nu}\right\} \tilde{g}, \quad\left\{\tilde{\Gamma}_{\mu}, \tilde{\Gamma}_{\nu}\right\}=2 \eta_{\mu \nu}-\tilde{g}\left\{g_{\mu}, g_{\nu}\right\}
$$

Introducing the time-like momentum $p^{\mu}\left(p_{\mu} p^{\mu}=m^{2}, m \neq 0\right)$, from (3.4) we get $\left(p^{\mu} \Gamma_{\mu}\right)^{2}=$ $m^{2}\left(1-\left(e^{\mu} g_{\mu}\right)^{2} \tilde{g}\right)$ and $\left(p^{\mu} \tilde{\Gamma}_{\mu}\right)^{2}=m^{2}\left(1-\tilde{g}\left(e^{\mu} g_{\mu}\right)^{2}\right)$ with $e^{\mu} \equiv p^{\mu} / m$. Below we shall shaw (see Eq. (3.5) ) that the operators $\left(1-\left(e^{\mu} g_{\mu}\right)^{2} \tilde{g}\right)$ and $\left(1-\tilde{g}\left(e^{\mu} g_{\mu}\right)^{2}\right)$ are the projectors onto the physical space for $\psi$ and $\bar{\psi}$, respectively. We call $\Gamma_{\mu}$ and $\tilde{\Gamma}_{\mu}$ the left and right almost Dirac matrices since being restricted to the physical subspaces, they satisfy a usual quadratic Clifford algebras. 


\subsection{Projectors}

To solve Eq. (3.2), we introduce two series of projectors (for $\psi$ and $\bar{\psi}$ ),

$$
\begin{array}{rlrlrl}
\Pi_{ \pm}(p) & =-\frac{1}{2}\left( \pm e^{\mu} g_{\mu}+\tilde{g}\right)^{2} \tilde{g} & \Pi_{ \pm}^{\star}(p) & =-\frac{1}{2} \tilde{g}\left( \pm e^{\mu} g_{\mu}+\tilde{g}\right)^{2} \\
& =\frac{1}{2}\left(\left(1-\left(e^{\mu} g_{\mu}\right)^{2} \tilde{g} \pm \tilde{g}^{2}\left(e^{\mu} g_{\mu}\right)\right),\right. & & & =\frac{1}{2}\left(\left(1-\tilde{g}\left(e^{\mu} g_{\mu}\right)^{2} \pm\left(e^{\mu} g_{\mu}\right) \tilde{g}^{2}\right),\right. \\
\Pi_{0}(p) & =\left(e^{\mu} g_{\mu}\right)^{2} \tilde{g}, & \Pi_{0}^{\star}(p) & =\tilde{g}\left(e^{\mu} g_{\mu}\right)^{2} .
\end{array}
$$

Using the outer automorphism $S O(d-1,1)$, we can consider $\Pi_{\varepsilon}(\hat{p})$ and $\Pi_{\varepsilon}^{\star}(\hat{p}), \varepsilon= \pm$, 0 , with $\hat{p}=(m, 0, \cdots, 0)$ the rest frame momentum. Then, it is easy to prove that $\Pi_{\varepsilon}(\hat{p}) \Pi_{\varepsilon^{\prime}}(\hat{p})=\delta_{\varepsilon \varepsilon^{\prime}} \Pi_{\varepsilon}(\hat{p})$ and $\Pi_{+}(\hat{p})+\Pi_{-}(\hat{p})+\Pi_{0}(\hat{p})=1$ (and similar relations for $\Pi^{\star}(\hat{p})$ ), i.e. $\Pi_{\varepsilon}(p)$ and $\Pi_{\varepsilon}^{\star}(p)$ constitute two complete sets of projectors. Denoting, for a given $k$-dimensional representation, $n_{\epsilon}=\operatorname{Tr}\left(\Pi_{\epsilon}(\hat{p})\right)=$ $\operatorname{Tr}\left(\Pi_{\epsilon}(p)\right)$, we have obviously $n_{+}+n_{-}+n_{0}=k$ and $n_{+}=n_{-}$. Moreover, using (2.3) we have $n_{0}=k / 3$ (this is enough to prove that $k$ is a multiple of 3 ), and finally we get

$$
\operatorname{Tr}\left(\Pi_{0}(p)\right)=\operatorname{Tr}\left(\Pi_{+}(p)\right)=\operatorname{Tr}\left(\Pi_{-}(p)\right)=n=\frac{1}{3} k,
$$

and similarly for $\Pi^{\star}$.

\subsection{Solutions to the equation $(3.2)$}

The projectors $\Pi$ and $\Pi^{\star}$ are useful to calculate the solutions to Eq. (3.2). From now on we just consider the equation for $\psi$, the other one is totally similar. Let us introduce the solutions of (3.2) in the form of the plane waves of positive and negative energy, $\psi_{ \pm}(p)=e^{\mp i p^{\mu} x_{\mu}} W_{ \pm}(p)$. Then, the equation is reduced to the equations for $W_{ \pm}(p),\left( \pm p^{\mu} g_{\mu}+m \tilde{g}\right) W_{ \pm}(p)=0$. From the relation $\left( \pm p^{\mu} g_{\mu}+m \tilde{g}\right)^{3}=m\left(p^{\mu} p_{\mu}-m^{2}\right)=0$, it is easy to see that $W_{ \pm}(p) \in \operatorname{Im}_{ \pm}(p)$. Finally, from $\left(p^{\mu} g_{\mu}\right)^{3}=0$ we observe that the set of vectors $W_{0}(p) \in \operatorname{Im} \Pi_{0}(p)$ are neither positive energy solutions nor negative energy ones, but the auxiliary fields. There the equations of motion are reduced to $W_{0}(p)=0$. Since, $\Pi_{\varepsilon}(p)$ constitute a complete set of projectors, the space of solutions decomposes into $S=\operatorname{Im} \Pi_{+}(p) \oplus \operatorname{Im} \Pi_{-}(p) \oplus \operatorname{Im} \Pi_{0}(p)$. The projectors $\Pi_{ \pm}(p)$ are related to the physical solutions, i. e. to the positive and negative energy solutions, whereas the projector $\Pi_{0}(p)$ characterizes the auxiliary fields. Differently to the quadratic Clifford algebra, here we have auxiliary fields (like they are present in generic Dirac-like equation (1.1)). This comes from the fact that the $g_{\mu}$ are singular, $\left(g_{\mu}\right)^{3}=0$. This singularity, in turn, is due to the fact that basically we have a quadratic form (related to the Minkowski norm) which we write through a 'cubic' form.

For the $d$-dimensional representation (2.11) the equation (3.2) takes a simple form. Writing $\psi^{t}=\left(\begin{array}{lll}\psi_{1} & \psi_{2} & \psi_{3}\end{array}\right)$, it gives

$$
\psi_{1}=0, \quad\left(i \gamma_{\mu} \partial_{\mu}+m \gamma_{d+1}\right) \psi_{i}=0, \quad i=2,3 .
$$

Hence, $\psi_{1}$ are auxiliary fermionic fields and $\psi_{2}, \psi_{3}$ are the usual Dirac fields.

For the representation (2.12), we have a relativistic wave equation in $2+1$ dimensions with Lorentz invariance only in the $\left(x_{0}, x_{1}\right)$ subspace. For the representation (2.14) in $1+1$ dimensions with a basis where $\Pi_{0}(\hat{p})=\operatorname{diag}(1,1,1,0,0,0,0,0,0)$, the Lorentz generator takes a simple form

$$
J=\frac{i}{3} \operatorname{diag}(1,1,1 ; 1,-2,1 ;-2,1,-2) .
$$

Therefore, it seems that the physical fields can be treated as $(1+1)$-dimensional anyons [18] of spin $1 / 3,-2 / 3$. We shall return to this point in last section. 


\subsection{Alternative calculation of Lorentz generators}

Assuming that for a given representation of $\langle g\rangle$ the Lorentz covariance of Eq. (3.2) is manifest, we have an alternative way to calculate the Lorentz generators. Indeed, we solve the equation (3.2) in any frame using the projectors $\Pi(p)$. Let us choose two particular frames: the rest frame and a frame such that $p^{\mu}=\left(p_{0} \sim m, p_{1} \ll m, 0, \cdots, 0\right)$. We denote respectively $W_{\varepsilon}(\hat{p})$ and $W_{\varepsilon}(p)$ the corresponding solutions, such that $S_{\varepsilon}(\hat{p})=\left\{W_{\varepsilon}^{i}(\hat{p}), i=1, \cdots, n\right\}$ and $S_{\varepsilon}(p)=\left\{W_{\varepsilon}^{i}(p), i=1, \cdots, n\right\}$ constitute a complete basis of solutions in these two frames. Now we have two ways to write $W_{\varepsilon}(p)$. On the one hand, we have

$$
W_{\varepsilon}(p)=C_{\varepsilon, p} \Pi_{\varepsilon}(p) W_{\varepsilon}(\hat{p}),
$$

with $C_{\varepsilon, p}$ a constant of normalization $\left(C_{\varepsilon, p} \sim 1\right.$, for $\left.p \sim \hat{p}\right)$. On the other hand, if we denote $B(p)$ the boost from the rest frame to the moving frame, we have

$$
W_{\varepsilon}(p)=B(p) W_{\varepsilon}(\hat{p}) .
$$

Comparing Eqs. (3.8) and (3.9), we obtain

$$
B(p)=\Pi_{+}(p) \Pi_{+}(\hat{p})+\Pi_{-}(p) \Pi_{-}(\hat{p})+\Pi_{0}(p) \Pi_{0}(\hat{p}) .
$$

For the infinitesimal boost along the $x_{1}$ axis, $\hat{p}^{\mu}=(m, 0, \cdots, 0) \longrightarrow p^{\mu}=(E \sim m, p \sim 0,0, \cdots, 0)$, developing (3.10) at the first order gives

$$
B(p)=1-i \frac{p}{m} J_{01}
$$

If we proceed along these lines for an arbitrary solution, $i$. e. with or without a manifest Lorentz covariance, the expression of $J_{01}$ (and in a similar way of $J_{0 i}$ ) looks not so symmetric (but at that point the algebra (2.3) is not used to simplify its expression). However, for the two solutions where the Lorentz covariance is guaranteed (like for representation (2.11) and $\mathcal{R}_{01}$ in $1+1$ dimensions), $J_{01}$ is reduced to a very simple expression (all other terms vanish):

$$
J_{01}^{p}=-\frac{i}{2}\left(\tilde{g} g_{1} g_{0}+g_{1} \tilde{g} g_{0}\right) .
$$

Compared with $J_{01}$ in (2.10), the term $-\frac{i}{2} g_{1} g_{0} \tilde{g}$ is missing. Obtaining $J_{01}^{p}$, we started from the equation (3.2) without any reference to the algebra (2.3). From the equation (3.2), if we do not make any reference to (2.3), only $\tilde{g}^{2} g_{\mu}$ (or $g_{\mu} \tilde{g}^{2}$ ) is a vector, but from the algebra (2.3) $g_{\mu}$ is a vector and $\tilde{g}$ is a scalar. It can be checked explicitly that $J_{\mu \nu}^{p}$ in (3.12) and $\tilde{g}^{2} g_{\mu}$ satisfy the correct commutation relations, although $g_{\mu}$ and $\tilde{g}$ with $J_{\mu \nu}^{p}$ do not. So, the generators $J_{\mu \nu}^{p}$ obtained in (3.12) can be called the partial generators and $J_{\mu \nu}$ in (2.10) the full ones. Even if we cannot obtain the full generators through this process, it gives some useful information. Indeed, in the previous section we have raised the possibility that $J_{\mu \nu}$ could (in principle) contain terms like $g_{\lambda} g^{\lambda}$. But such terms never appear with (3.10), and this supports the conjecture (on the form of $J_{\mu \nu}$ ) of the previous section.

\section{5 $\mathrm{U}(1)$ interaction}

Let us consider the problem of coupling the field system described by the cubic root equation (3.2) to the external $\mathrm{U}(1)$ gauge field $A_{\mu}$. Introducing the covariant derivative $\nabla_{\mu}=\partial_{\mu}-i e A_{\mu}$ (e being the charge of the field $\psi$ ), the equation becomes

$$
\left(i g^{\mu} \nabla_{\mu}+m \tilde{g}\right) \psi=0 .
$$


The calculation of $\left(i g^{\mu} \nabla_{\mu}+m \tilde{g}\right)^{3}$ gives

$$
\left(i g^{\mu} \nabla_{\mu}+m \tilde{g}\right)^{3}=-i\left(g_{\mu} \nabla^{\mu}\right)^{3}-m\left(\nabla_{\mu} \nabla^{\mu}+m^{2}-e F^{\mu \nu} J_{\mu \nu}\right)
$$

with $F_{\mu \nu}=\partial_{\mu} A_{\nu}-\partial_{\nu} A_{\mu}$, and $J_{\mu \nu}$ given by (2.10). Due to the algebra of generators (2.3), the first term from Eq. (3.14) is reduced to

$$
-i\left(g_{\mu} \nabla^{\mu}\right)^{3}=-\frac{1}{4} e\left(F^{\mu \nu} \nabla^{\lambda}\left(g_{[\mu} g_{\nu]} g_{\lambda}+g_{[\mu} g_{\lambda} g_{\nu]}+g_{\lambda} g_{[\mu} g_{\nu]}\right)-e \mathcal{T}(A), \mathcal{T}=\left(\partial^{\mu} \partial^{\nu} A^{\lambda}\right) g_{\mu} g_{\nu} g_{\lambda} .\right.
$$

The last term in Eq. (3.15) seems to be not manifestly gauge-invariant, but its change under the gauge transformation $A_{\mu} \rightarrow A_{\mu}+\partial_{\mu} \Lambda$ disappears due to the algebra (2.3): $\partial_{\mu} \partial_{\nu} \partial_{\lambda} \Lambda g^{\mu} g^{\nu} g^{\lambda}=0$. With this observation the term $\mathcal{T}$ can be transformed identically due to the algebra (2.3) into the manifestly gauge-invariant form $\mathcal{T}=\frac{1}{6} \partial_{\lambda} F_{\mu \nu}\left(g^{\lambda} g^{[\mu} g^{\nu]}+g^{[\mu} g^{\lambda} g^{\nu]}\right)$. Summarizing all this, we conclude that the modified Klein-Gordon equation corresponding to our field system interacting with the U(1) gauge field via the prescription of minimal coupling (3.13) is given by

$$
\begin{aligned}
\left(\nabla_{\mu} \nabla^{\mu}+m^{2}\right. & -e F^{\mu \nu} J_{\mu \nu} \\
& +\frac{e}{2 m} F^{\mu \nu}\left(g_{\lambda} g_{\mu} g_{\nu}+g_{\mu} g_{\lambda} g_{\nu}+g_{\mu} g_{\nu} g_{\lambda}\right) \nabla^{\lambda} \\
& \left.+\frac{e}{3 m} \partial^{\lambda} F^{\mu \nu}\left(g_{\lambda} g_{\mu} g_{\nu}+g_{\mu} g_{\lambda} g_{\nu}\right)\right) \psi=0 .
\end{aligned}
$$

Therefore, besides the standard spin-field coupling described by the third term in modified KleinGordon equation (which appears in the case of Dirac field with minimal coupling prescription), here in generic case we have new structures given by the 4 -th and 5 -th terms in Eq. (3.16). But direct checking shows that in representations (2.11) and (2.14), for which Lorentz symmetry is manifest, the relation $g_{\mu} g_{\nu} g_{\lambda}=0$ is valid. Therefore, in these cases the modified Klein-Gordon equation has the same structure as the quadratic equation corresponding to Dirac equation with minimal coupling. This means, in particular, that the corresponding Lorentz covariant field systems are characterized by the gyromagnetic ratio $g=2$.

In the peculiar $(1+1)$-dimensional case (2.14), because of the 'renormalization' of (2.10), the equation takes the form

$$
\left(\nabla_{\mu} \nabla^{\mu}+m^{2}-e F^{01} J_{01}-\frac{e}{3} F^{01}\right) \psi=0
$$

with $J_{01}$ given by 2.15 .

\section{Concluding remarks}

Clifford and Grassmann algebras are the basic ingredient for the spinorial representation of the Lorentz group and they naturally appear as fundamental generators for the Dirac operator. In this paper, we have constructed a new wave equation in line of Dirac equation. Indeed, from Clifford algebra of a polynomial of degree higher than two (cubic in our case) we were able to define a relativistic wave equation involving the cubic root of the Klein-Gordon operator instead of a square root. Moreover, as Dirac equation is related to supersymmetry, it has been observed that the equation considered in this paper can be related to an extension of supersymmetry involving an $n$-th order algebra, namely fractional supersymmetry [16, 17]. Conversely, it is well known that supersymmetry is related to Clifford and Grassmann algebra. Similarly it has also been established that fractional supersymmetry is connected to Clifford algebras of polynomials [15]. So, all these 
$n$ order structures (Clifford algebra of polynomials, fractional supersymmetry and $n$-th root of the Klein-Gordon operator) are interconnected.

On a more practical ground, within the framework of the Clifford algebra of the polynomial $m\left(p_{\mu} p^{\mu}-m^{2}\right)$ the relativistic equation generalizing Dirac equation has been obtained. Differently to the quadratic case, where the spin content is unique (spin-1/2 particles), here the spin content is related to the representation of the Clifford algebra we take. Only finite dimensional representations are known for this type of algebras. Two explicit matrix representations are given here, and one of them gives an appropriate equation for $(1+1)$-dimensional anyons of spin $1 / 3$ and $-2 / 3$.

Constructing the corresponding Lorentz generator for the $1+1$ anyonic case, we fixed the corresponding matrix to be traceless. So, strictly speaking, the question on spin content of such anyonic-like fields deserves further investigation. This point can be clarified by realizing secondary quantization to reveal the corresponding spin-statistics relation. Since the massive field theory in $1+1$ dimensions can be treated as a reduction of the corresponding massless theory in $2+1$ dimensions, here the analysis of ref. [19] on statistics for massless $(2+1)$-dimensional theories may be very helpful.

No infinite dimensional representation of the infinite Clifford algebra $\langle g\rangle$ has been found here. It would be very interesting to construct such representations in the context of $(2+1)$-dimensional anyons.

Finally, we could have chosen a slightly different cubic Clifford algebra, for which the massive and massless cases would have been put on the same footing. Introducing some universal mass parameter $M$ (it could be, e.g., the Planck mass), one can consider the Clifford algebra $M\left(p_{\mu} p^{\mu}-m^{2}\right)=$ $\left(p_{\mu} g^{\mu}+m \tilde{g}+M \hat{g}\right)^{3}$.

Acknowledgements Ph. Revoy is gratefully acknowledged for useful discussions. One of us (MRT) would like to thank USACH for its hospitality, where the part of this work was realized. The work was supported in part by the grants 1980619 and 7980044 from FONDECYT (Chile) and by DICYT (USACH).

\section{References}

[1] G. Petiau, Acad. Roy. de Belg., A. Sci. Mem. Collect 16 (1936); R. Y. Duffin, Phys. Rev. 54 (1938) 1114; N. Kemmer, Proc. Roy. Soc. A173 (1939) 91.

[2] H. S. Green, Phys. Rev. 90 (1953) 270; O. W. Greenberg, Phys. Rev. Lett. 13 (1964) 598; O. W. Greenberg and A. M. L. Messiah, Phys. Rev. B136 (1964) 248; B138 (1965) 1155; Y. Ohnuki and S. Kamefuchi, Quantum Field Theory and Parastatistics (Springer Verlag, 1982).

[3] M. S. Plyushchay, Phys. Lett. B262 (1991) 71; Nucl. Phys. B362 (1991) 54.

[4] J. L. Cortés and M. S. Plyushchay, Int. J. Mod. Phys. A11 (1996) 3331, hep-th/9505117 and references therein.

[5] R. Jackiw and V. P. Nair, Phys. Rev. D43 (1991) 1933.

[6] M. S. Plyushchay, Phys. Lett. B273 (1991) 250; Phys. Lett. B320 (1994) 91, hep-th/9309148;

J. L. Cortés and M. S. Plyushchay, J. Math. Phys. 35 (1994) 6049, hep-th/9405193.

[7] M. S. Plyushchay, Nucl. Phys. B491 (1997) 619, hep-th/9701091. 
[8] M. S. Plyushchay, Mod. Phys. Lett. A12 (1997) 1153, hep-th/9705034.

[9] R. Jackiw and S. Templeton, Phys. Rev. D23 (1981) 2291; J. Schonfeld, Nucl Phys. B185 (1981) 157; S. Deser, R. Jackiw and S. Templeton, Phys. Rev. Lett. 48 (1982) 975.

[10] V. I. Fushchich and A. G. Nikitin, Symmetry of Quantum Mechanical Equations (Moscow, Nauka, 1990) [in Russian].

[11] N. Roby, C. R. Acad. Sc. Paris 268 (1969) 484; N. Roby, Bull. Sc. Math. 94 (1970) 49; Ph. Revoy, C. R. Acad. Sc. Paris 284 (1977) 985.

[12] N. Fleury, M. Rausch de Traubenberg, J. Math. Phys. 33 (1992) 3356; N. Fleury and M. Rausch de Traubenberg, Adv. Appl. Cliff. Alg. 4 (1994) 123.

[13] Ph. Revoy, Adv. Appl. Cliff. Alg. 3 (1993) 39.

[14] D. H. Haile and S. Tesser, J. Alg. 116 (1988) 372.

[15] M. Rausch de Traubenberg, Habilitation Thesis, hep-th/9802141 [in French].

[16] N. Fleury and M. Rausch de Traubenberg, Mod. Phys. Lett. A11 (1996) 899, hep-th/9510108.

[17] M. Rausch de Traubenberg and M. J. Slupinski, hep-th/9904126.

[18] J. Gamboa and J. Zanelli, Phys. Lett. B357 (1995) 131, hep-th/9503126.

[19] S. Deser and R. Jackiw, Phys. Lett. B263 (1991) 431. 